\title{
Vita Nuova, XIX. Intorno a una traduzione di Joan Maragall ${ }^{1}$
}

\section{Francesco Ardolino}

Universitat de Barcelona

\begin{abstract}
Il ritrovamento del manoscritto di una traduzione maragalliana della Canzone di Vita Nuova, XIX getta una nuova luce sul dantismo di Maragall e aiuta a dipanare alcuni punti oscuri su una versione pubblicata nella rivista Lletra e non raccolta nelle Obres Completes. Nel presente studio si inquadrerà la versione maragalliana all'interno del preraffaellitismo catalano, si analizzeranno le soluzioni testuali proposte dalllo scrittore catalano e si genererà una proposta di edizione.
\end{abstract}

Parole chiave: traduzione, ricezione, Dante e dantismo, Maragall, Catalogna.

\section{Abstract}

The rediscovery of the manuscript containing a translation by Maragall of Vita Nuova XIX sheds new light on Maragall's Danteism and helps in clarifying certain obscure points in a version published by the journal Lletra, which never formed part of the Obres Completes. The current study places Maragall's version within the environment of the Catalan preRaphaelite movement, examines the textual solutions put forward by the Catalan writer and sets out proposals for editing the work.

Key words: translation, reception, Dante and Danteism, Maragall, Catalonia.

1. Quest'articolo rientra nel «Proyecto de investigación Boscán», BFF 2002-01860, Bibliografia textual aplicada a las traducciones españolas de la literatura italiana (catálogo automatizado de las traducciones literarias en castellano y catalán: 1400-1939), coordinato dalla Prof.ssa María de las Nieves Muñiz Muñiz. In particolare, si tratta della versione italiana dell'ultimo capitolo di un lavoro dal titolo (provvisorio) «Prosa, poesia poètica i traducció: Dante a l'obra de Joan Maragall». Con le sigle OC I e OC II si indicheranno i due volumi di Joan Maragall, Obres Completes, Barcellona: Selecta, 1981 [1960 e 1961], dedicati, rispettivamente, alla produzione in lingua catalana e a quella in spagnolo. 


\section{Presentazione}

In una copia della traduzione catalana della Vita Nuova ad opera di Manuel de Montoliu conservata nella Biblioteca di Filologia dell'Universitat de Barcelona, si possono leggere ai margini vari appunti, con ogni probabilità risalenti alla mano del curatore, sparsi lungo tutto il testo. ${ }^{2}$ Il chiosatore si sfoga, in particolare, intorno alla canzone «Oh dònes, que capiu ço que Amor sia». ${ }^{3}$ Nel 1937, la versione di Montoliu verrà sottoposta a normalizzazione ma non saranno introdotte nelle note le idee segnate a penna su questa copia del libro. ${ }^{4}$

2. Dant Allighieri, La Vida Nova, Barcellona: Biblioteca Popular de «L'Avenç», 1903. La notizia del ritrovamento di un esemplare con note autografe era già stata annunciata da Gabriella GAVAGNIN, La letteratura italiana nella cultura catalana (1918-1936), tesi di dottorato, Universitat de Barcelona, p. 244 e 247. La firma a mo’ di ex libris che Montoliu lascia nella prima di copertina combacia - almeno nell'analisi di un principiante assoluto in perizie calligrafiche, come l'autore di quest'articolo — con la scrittura usata negli appunti interni al libro.

3. La traduzione occupa le pagine 47-49 (rispettivamente v. 1-23, 24-51 e 52-70). Alla fine dell'invocazione dell'Angelo al Signore, nella seconda strofa, c'è un rimando a nota dove si può leggere "La terra no es digna de Beatriu. V. Influencias Platónicas». Alla fine della terza strofa ("I encar li ha Déu major mercè donat, / che no pot mal morir qui li ha parlat»), con un altro rimando si enuncia "Recort del culte à la Verge Maria. Poco più sotto (v. 43-44), la domanda d'Amore, "Com es que mortal cosa / tant ornada pot esser i tant pura?», è glossata come una conferma di quanto detto sopra: «Igual». La pagina 48 è percorsa verticalmente da due scritte: nella parte più esterna si legge "Personificacions de la bontat divina» $e$, in quella interna, "El Dant posa en Beatriu una sintesi de totes les virtuts secretes y miraculoses que '1 Cristianisme atribueix als seus sants".

4. Dante Allighieri, La Vida Nova, Barcellona: Edicions de la Rosa dels Vents, 1937. Non va, invece, presa in considerazione la nuova edizione - DANTE, Vida nova, Barcellona: Quaderns Crema, 1999 - curata (si fa per dire) da Jaume Vallcorba. L'editore, infatti, impone di suo pugno la «revisió, presentació i notes complementàries» seguendo un bizzarro criterio di rispetto dell'intentio auctoris: «autoritzat per la nota a l'edició de 1937, segons la qual Manuel de Montoliu va suggerir una revisió i regularització de l'original a favor de l'"expressió normal del català literari viu" ", he passat a perifràstiques les primeres persones del pretèrit perfect dels textos en prosa, $\mathrm{i}$ he substituït unes quantes - molt poquesparaules»; cfr. Jaume VAlLCORBA, «Nota editorial», ibid., p. 15-16 (cit., p. 15). Non male, per un'edizione in cui 1) la «Nota editorial» del 1937 non appare poiché, se apparisse, i nodi verrebbero subito al pettine. Eccola qui: «N. de la D.: Hem volgut trascriure íntegrament aquest pròleg, a desgrat del seu parcial desplaçament — cal recordar que del 1903 ençà la nostra llengua ha arribat a un recobrament absolut — perquè l'estudi que s'hi fa de l'obra traduïda il-lustrarà bellament el lector. Ens cal afegir, només, que, volent honorar la confiança que deposità en nosaltres el senyor de Montoliu, autoritzant-nos a revisar i a normalitzar l'original, ens hem limitat a una correcció estrictament ortogràfica, i en alguns casos, en els versos, a resoldre'ls donant a l'expressió literària la forma més aproximada a l'expressió del català viu». In cui 2) mentre nella «Nota editorial» vallcorbiana si annuncia que «el text que avui presentem reprodueix en part el d'aquesta reimpressió [de 1937] esmenat a la vista de la primera, al qual s'han fet correccions i addicions tenint al davant l'edició crítica de Michele Barbi» (cit., p. 15), nella quarta di copertina si afferma tutto il contrario: «per la present edició hem recuperat la traducció catalana que en va fer Manuel de Montoliu [...] el 1903, revisada a la vista de les més solvents edicions modernes». Ma si vedano solo le varianti relative al primo paragrafo nelle tre edizioni (si elimina il rimando a nota e si risaltano le divergenze in grassetto): MONTOLIU 1903: «En aquella part del llibre de la meva memòria, abans de la qual poc s'hi podria llegir, s'hi troba una rúbrica, la qual diu: Incipit 
L'interesse per la Vita Nuova, da parte del preraffaellismo catalano a cavallo tra i due secoli, è vivissimo e il fatto che Montoliu acconsentisse alla correzione della grammatica e dell'ortografia del suo testo per farlo entrare in sintonia con i tempi, è più che comprensibile; piuttosto, sarà da riconoscere l'impegno profuso dallo studioso, nell'arco di almeno quattro decenni, nell'esegesi del testo. $^{5}$

$\grave{E}$ al centro di questo entusiasmo - da cui era stato catturato non solo Montoliu ma tutto un gruppo di intellettuali catalani, Modernisti e Noucentisti - che va collocato il tentativo di traduzione operato da Maragall della canzone qui in esame.

Prima di dedicare la nostra attenzione alla versione di Maragall, varrà la pena affrontare due questioni di fondo, che potranno sembrare opposte ma che, in un discorso critico globale, diventano complementari. La prima è l'interesse del Poeta verso le traduzioni in catalano; la seconda riguarda la mancata conoscenza e il successivo disinteresse dimostrato dagli studiosi maragalliani nei confronti di questa traduzione.

Per quanto concerne il primo punto, il Nostro non vacilla mai nelle sue affermazioni e mantiene legato il concetto di amore per la lingua (catalana) a

Vita Nova. Sota la qual rúbrica, jo trobo escrites les paraules que tinc el pensament de retreure en aquest llibret; i, si no totes, al menys la llur significança»; MONTOLIU 1937: «[...] poc es podria llegir, es troba una rúbrica que diu: Incipit Vita Nova. Sota la qual trobo escrites les paraules [...] al menys la seva significança»; VALLCORBA-MONTOLIU 1999: «[...] poc s'hi podria llegir, hi ha una rúbrica que diu: Incipit vita nova. Sota aquesta rúbrica trobo escrites les paraules [...] almenys la seva significança». Si dia la parola all'edizione Barbi, chiamata in causa da Vallcorba, come appare riprodotta nell'Appendice dell'Enciclopedia dantesca: «In quella parte del libro de la mia memoria dinanzi a la quale poco si potrebbe leggere, si trova una rubrica la quale dice: Incipit vita nova. Sotto la quale rubrica io trovo scritte le parole le quali è mio intendimento d'assemplare in questo libello; e, se non tutte, almeno la loro sentenza». Quindi, Vallcorba recupera la parola «rúbrica», presumibilmente sfogliando il testo italiano, ma non la ripetizione dantesca del verbo «trovare», forse perché non la trovava abbastanza elegante. Infine, siamo di fronte a un'edizione in cui 3) anche il titolo è stato cambiato, visto che l'articolo «la» era presente sia nell'edizione del 1903 che in quella del 1937, ma la decapitazione avviene impietosamente sotto la scure dei Quaderns Crema. Vallcorba non è nuovo a operazioni di questo genere, che potremmo battezzare come nuovo approccio filologico basato sul principio "L'auteur, c'est (à) moi»: ne dà fede anche in una polemica, riguardante l'edizione delle opere foixiane, con Veny-Mesquida nelle pagine della rivista Els Marges; cfr. Joan Ramon VENY-MESQUIDA, «Sobre l'edició crítica de textos», Els Marges, n. 67, ottobre 2000, p. 75-107; J. VALLCORBA, «Sobre l'edició de textos: a propòsit d'una nota de Veny-Mesquida i les edicions de Foix", Els Marges, n. 68, dicembre 2000, p. 117-121; J. R. VENY-MESQUIDA, «Encara sobre els textos de J. V. Foix publicats a Quaderns Crema. (Resposta a l'editor Jaume Vallcorba)", Els Marges, n. 69, gennaio 2001, p. 96-111. Su un aspetto che ci tocca più da vicino, bisognerà aspettare uno studioso che, pazientemente, controlli i cambiamenti imposti dal Modernizzatore alla nuova edizione della traduzione di Sagarra della Divina Commedia.

5. Gavagnin analizza in modo particolareggiatissimo le operazioni culturali condotte in Catalogna nell'ambito del sesto centenario della morte di Dante. Tra esse, c'è un ulteriore intervento di Montoliu ma, malgrado i mutamenti ideologici dell'autore messi in luce da Gavagnin, non si trovano modifiche sostanziali alla linea che aveva più volte sostenuto nel primo decennio del Novecento; cfr. G. GAVAGNIN, op. cit., p. 244-248. 
quello del rafforzamento culturale di essa attraverso l'incorporamento degli altri testi letterari mediante le traduzioni. Vediamone un paio di esempi:

I una llengua que, tenint prou força assimiladora, se troba en les condicions que avui se troba la catalana, jo crec que també es pot aprofitar molt d'un altre element de correu que és la traducció de les grans obres literàries estrangeres. El treball de traducció, quan és fet amb calor artístic, suggereix formes noves; fa descobrir riqueses de l'idioma desconegudes, li dóna tremp i flexibilitat, el dignifica per l'altura de lo traduït, i en gran part li supleix la falta d'una tradició literària pròpia i seguida. De més, el posa en contacte amb l'esperit humà universal i li fa seguir la marxa amb ell. ${ }^{6}$

Así al lado de la resurreción de nuestros clásicos debemos espaciarnos en la traducción de las mejores obras de las literaturas extranjeras adaptándolas a nuestra expresión, que es como adaptar ésta a lo universal del espíritu humano. ${ }^{7}$

Insomma, la traduzione rappresenta un preciso percorso politico-culturale per l'edificazione di una Catalogna nazionale — o regionale che sia. Il «despert entre adormits» è lungimirante e, dando uno sguardo alla sua produzione, non lo si può certo accusare di predicare bene e razzolare male.

Cal atorgar a Maragall el mèrit d'haver reconegut la importància que tenia per al desenvolupament del català el contacte amb les altres literatures. El seu impetuós i juvenil contacte amb l'obra de Goethe fou el començament d'un camí ric de conseqüències per a la seva formació literària i per a l'enriquiment del seu estil i simultàniament de la llengua catalana en general. ${ }^{8}$

Passando in rassegna le versioni di Maragall, si scoprono sistematicamente gli altarini dei suoi autori prediletti: è per questo che il rinvenimento di una traduzione di Dante avrebbe potuto contribuire a spostare il baricentro degli interessi maragalliani in una zona finora poco o punto esplorata dai critici. Il documento che si riproduce nelle pagine seguenti è la prova del nove di quella funzione-Dante che, in altro luogo, si ipotizzava come possibile.

Quest'ultima osservazione si lega alla seconda ragione di fondo. La versione maragalliana non è inedita: il testo venne pubblicato nel primo numero della rivista Lletres di Girona ma, nelle Obres Completes, nei vari saggi critici su Maragall, persino nelle bibliografie specializzate non se ne faceva menzione. $\grave{E}$ quindi con giusto risalto che Fulcarà, in uno studio sul modernismo gerundino, riporta la traduzione della canzone con un sopratitolo altisonante, «Un text desconegut de Joan Maragall», e segnala, in nota, che «l'aparició d'un text inèdit de Joan Maragall a les pàgines de Lletres és un fet indicatiu de la quali-

6. «El catalanisme en el llenguatge. Fragments d'un discurs» (1893), in OC I, p. 789.

7. "Traducciones», Diario de Barcelona, 5-12-1901, in OC II, p. 166.

8. Cfr. Jaume Tur, Maragall $i$ Goethe. Les traduccions del «Faust», Departament de Filologia Catalana de la Universitat de Barcelona, 1974, p. 57. 
tat de la publicació». ${ }^{9}$ La rivista, che aveva visto la luce nell'aprile del 1907 , arrivò fino al numero nove, rispettando per lo più la scadenza mensile e chiudendo definitivamente i battenti a novembre dello stesso anno. ${ }^{10}$ Anche Joaquim Molas, autore del prologo al libro di Fulcarà, si rende conto del valore della scoperta e, nel momento di tessere le lodi allo studio che segue, avverte che vi si troveranno degli apporti decisivi e che l'autrice «dóna a conèixer, per exemple, una traducció maragalliana desconeguda d'una de les obres centrals del Modernisme i fins del Noucentisme: la Vita nuova». ${ }^{11}$

Fin qui tutto rientrerebbe nei termini di un ritrovamento seguito a una perdita "per distrazione» e il dato non susciterebbe alcuno scalpore. Il fatto è, però, che la traduzione in questione è di nuovo caduta nell'oblio e gli specialisti maragalliani non la citano e, forse, neppure la conoscono. Dall'altra parte, non è che le cose vadano tanto meglio ed è sintomatico che Rossend Arqués, che nel suo saggio sul dantismo catalano dedica alcune pagine intuitive - di un'intuizione valida e proficua - a Maragall, non ne faccia menzione. ${ }^{12}$

Eppure, è lo stesso Maragall a ringraziare epistolarmente Carles Rahola della rivista appena ricevuta e a chiamare in causa il proprio contributo; tra l'altro, in una lettera precedente aveva accolto possibilisticamente l'idea di partecipare con qualcosa di suo pur senza specificarne le caratteristiche. ${ }^{13}$ Certo, non indicava titoli né nomi, ma dava tutte le informazioni per reperire un documento che sembrava scritto per l'occasione e che, perciò, aveva tutte le carte in regola per far sospettare della sua originalità.

Non è ancora finita. Nell'Arxiu Maragall si trova infatti conservato un foglio di dimensioni ridotte $(24,5 \times 15 \mathrm{~cm})$ scritto sulle due facciate con il testo a uno stadio molto simile a quello della traduzione pubblicata nella rivista. ${ }^{14}$ Qui di seguito, si offre la versione apparsa in Lletres, con le varianti del manoscritto e le relative correzioni autografe. Il testo italiano a fronte è, invece, quello contenuto nel volume di Fraticelli presente nella biblioteca privata di

9. Cfr. Maria Dolors FulCarà i Torroella, Girona i el modernisme, Girona: Instituto de Estudios Gerundenses, 1976, p. 169.

10. La rivista è consultabile presso la Biblioteca Pública di Girona. Per una descrizione esaustiva, cfr. ancora M. Dolors FulCARÀ I TORROELla, op. cit., p. 76-81.

11. Joaquim Molas, «Pròleg», in M. Dolors FulCarÀ I TORROElla, op. cit., p. VIII.

12. Cfr. Rossend ARQuÉs, «El rastro de la pantera perfumada (Dante en las poéticas de la modernidad)», Tenzone, n. 1, 2000, p. 179-214. Ora in catalano con il titolo «El rastre de la pantera perfumada (Dante en les poètiques de la modernitat)", Quaderns de la Fundació Maragall, n. 53, 2000 [febbraio 2001], p. 23-53. La notizia è invece raccolta da Assumpta CAMPS, «El Dante de La vita nova en català», Revista de Catalunya, n. 122, ottobre 1997, p. 89102 (97) e, successivamente, sviluppata in A. CAMPS, "De una canción de La Vita Nova de Dante Alighieri traducida por Joan Maragall», Voz y Letra, tomo VIII, vol. 1, 1997, p. 5-12; ne dimostra la conoscenza anche Lluís QUINTANA TRIAS, La veu misteriosa, Barcellona: Publicacions de l'Abadia de Montserrat, p. 321.

13. Le lettere, la prima dell'1-3-1907 e l'altra senza data, si trovano in OC I, p. 1076-1077. Il riferimento è naturalmente già stato indicato sia da Fulcarà che da Camps.

14. Attraverso il foglio molto sottile si legge in filigrana il numero "4800». Il documento era stato catalogato con un errore di trascrizione nello schedario manuale dove risulta l'intestazione "Diuen que haveu enteniment d'amor». 
Maragall. ${ }^{15}$ Si lasciano quindi in coda l'edizione critica del testo e il commento relativo.

\section{Il testo pubblicato e le varianti autografe $\mathrm{e}^{16}$}

\section{D'una cansó de la Vida Nova del Dante Alighieri \\ (fragments traduhits per J. Maragall)}

v. $1-8$

Dones que haveu enteniment d'amor Vull ab vosaltres de la meva dir No perque crega sua llahor finir Sino parlar per desfogâ el meu cor. Vos diré que pensant el seu valor Amor se 'm fá tant dolçament sentir Que si no fos llavors mon defallir Parlant faría enamorar tot cor v. $1-14$

Donne ch'avete intelletto d'amore, Io vo' con voi della mia donna dire; Non perch'io creda sua laude finire, Ma ragionar per isfogar la mente. Io dico che pensando il suo valore, Amor sì dolce mi si fa sentire, Che, s'io allora non perdessi ardire, Farei, parlando, innamorar la gente: Ed io non vo' parlar sì altamente, Ch'io divenissi per temenza vile; Ma tratterò del suo stato gentile A rispetto di lei leggeramente, Donne e donzelle amorose con vui, Chè non è cosa da parlare altrui.

2) Ms: dela. 3) Ms: llaor. 4) Ms: Mes raonar per desfogar el meu cor; CMs: desfogâ. 6) Ms: fa. 8) Ms: faria.

\section{v. $9-22$}

Un ángel clama ab divinal clamor Y diu: «Senyor, al mon s' hi esdevé Una gran meravella que provê D' esprit que fins aquí llença claror. Y tot el cel, al qui no manca rê Sino haverlo, demána'l al Senyô, Y tots els Sants ne volen la mercè:

\section{v. $15-28$}

Angelo clama in divino intelletto E dice: Sire, nel mondo si vede Meraviglia nell'atto, che procede $\mathrm{Da}$ un' anima, che fin quassù risplende: Lo Cielo che non have altro difetto Che d'aver lei, al suo Signor la chiede; E ciascun santo ne grida mercede.

15. Dante Alighieri, Opere minori, a cura di Pietro Fraticelli, Napoli: Francesco Rossi-Romano, 1855.

16. Legenda: $\mathrm{Ms}=$ testo base del manoscritto; $\mathrm{CMs}=$ correzioni successive sul manoscritto. Il testo base è quello pubblicato nella rivista Lletres. 
No mes pietat ens dona la rahó.

Que Deu ohint que 's parla d' ella: «Amats»

—Diu á ne 'ls sants_ « «no vos despacientèu,

Que allá ont ella es hi há algú que quan

l' haureu

$\mathrm{Al}$ infern anirá á dî als condemnats:

- Jo he vist la esperança dels benaven-

turats-》

¡Ma dona es desitjada á dalt del cel!
Sola pietà nostra parte difende;

Chè parla Iddio, che di madonna intende:

Diletti miei, or sofferite in pace,

Che vostra speme sia quanto mi piace

Là ov'è alcun che perder lei s'attende

E che dirà nello inferno a' malnati:

Io vidi la speranza de' beati.

9) Ms: on [?] dur el; CMs: ab divinal clamor. 13) Ms: Y tot el Cel que sols trova mancança; CMs: al qui no manca res; CMs2: rê. 14) Ms: D'aquest esprit; CMs: Sino haverlo. Ms: Senyor. 17) Ms: d'ella diu:; CMs: d'ella: «Amats: 18) Ms: Estimats; CMs: —Diu nels Sants_. Ms: despacienteu. 19) Ms: alla. que la perdra; CMs: quan l'haureu. 19bis) Ms: Que'm plau que ont es hi ha algù que quan l'haureu [cassato in blu]. 20) Ms: que al infern anira à; CMs: al infern podrà anâ à; 21) Ms: Jo hè vist a [?] esperança [cassato in blu]; CMs: Jo he vist la esperança. 22) Ms: à.

\section{v. $23-34$}

Ara os diré quina es la sua virtut:

Dòna que vulla apendre gentilesa,

Que vaja ab ella, que quan vá fent vía

En els homes grollers posa una feresa

Que aquell que baixament la miraría

Oíen seria ennoblit ó 's moriría.

Mes quan ne troba algún que digne sía

De mirarla, en aquell té tal virtut

Que se sent molt mellor de lo que 's pensa,

Y tant humil, que oblida tota ofensa.

Y encara major gracia Deu li há dat:

Que no pot mal finir qui li há parlat v. $29-42$

Madonna è desiata in sommo cielo:

Or vo' di sua virtù farvi sapere:

Dico: qual vuol gentil donna sapere

Vada con lei, che quando va per via,

Gitta ne' cor villani Amore un gelo

Per che ogni lor pensiero agghiaccia e pere:

E qual soffrisse di starla a vedere

Diverria nobil cosa, o si morria:

E quando trova alcun che degno sia

Di veder lei, quei prova sua virtute,

Chè gli addivien ciò che gli dà salute

E sì l'umilia, che ogni offesa oblìa

Ancor le ha Dio per maggior grazia dato

Che non può mal finir chi le ha parlato.

24) Ms: Dona. 25) Ms: và fent vía. 26) Ms: En els cors. CMs: En els homes. Ms: posa feresa; CMs: posa una feresa. 27) Ms: miraria. 29) Ms: Ò en seria — ò ‘s moriria. 29) Ms: algun. 33) Ms: li hà dat. 34) Ms: li hà parlat. 


\section{v. $35-44$}

Color de perla, que à bell rostre escau, Es el seu; esblaymat, pero no massa; Es tot lo bé que puga fer natura Y d' humana bellesa la mesura. Y dels ulls ván sortintli mentres passa Uns esperits d' amor tots inflamats Que fereixen els ulls del qui la mira Y van de dret al cor; y dû estragit Amor meteix al rostre: tal el gira Que no pot pas mirarse fit á fit.

\section{v. $43-56$}

Dice di lei Amor: cosa mortale Come esser può sì adorna e sì pura? Poi la riguarda, e fra sè stesso giura Che Dio ne intende di far cosa nova. Color di perla quasi informe, quale Conviene a donna aver non fuor misura: Ella è quanto di ben può far natura; Per esempio di lei beltà si prova. Degli occhi suoi, comecch'ella gli muove, Escono spirti d'amore infiammati Che fieron gli occhi a qual, che allor gli guati,

E passan sì che 'l cor ciascun ritrova: Voi le vedete Amor pinto nel viso, Ove non puote alcun mirarla fiso.

35) Ms: que à bell; CMs: que à un bell. 36) Ms: Es del; CMs: Es el seu. 39) Ms: van. 42) Ms: vàn - dù. 43) Ms: aixís es dit; CMs: tal el gira.

\section{Proposta d'edizione ${ }^{17}$}

Dones que haveu enteniment d'amor

Vull amb vosaltres de la meva dir

No perquè crega sua llaor finir

Sinó parlar per desfogar el meu cor.

Vos diré que pensant el seu valor

Amor se'm fa tan dolçament sentir

Que si no fos llavors mon defallir

Parlant faria enamorar tot cor.

\section{Un àngel clama amb divinal clamor}

I diu: «Senyor, al mon s’hi esdevé

17. Per quanto riguarda i criteri di edizione, si sono volute rispettare la maggior parte delle norme sistematizzate da Glòria Casals nella sua edizione critica dei libri di poesia pubblicati in vita da Maragall; cfr. Glòria Casals, "Criteris d'edició», in J. Maragall, Poesia, Barcellona: La Magrana, 1997, p. 61-65. Pertanto, si è modernizzato l'uso degli accenti e di alcune forme grafiche concomitanti $(i$ per $y)$; si è adeguata la punteggiatura nel modo più conservativo possibile; si sono risolte in amb e in on le forme $a b$ e ont maragalliane oltre alla riduzione a tan della forma tant con efelcistica davanti ad avverbio e aggettivo; si sono mantenute le elisioni del pronome es in posizione atona ed enclitica preceduta da vocale; si è sempre restituita la $-r$ elisa, anche quando segnalava evidentemente una sinalefe ( $d \hat{u}$ estragit) comunque facilmente interpretabile, prendendo le distanze in questo specifico caso da quanto proposto da Casals per evitare un surplus di accentazioni grafiche. Si è mantenuta invece l'alternanza tra le forme espritlesperit, facilmente spiegabile per ritmo e computo metrico, ma anche quella algúlalgun, e non si è proceduto all'apostrofazione quando implicava un'elisione di vocale (la esperança). 
Una gran meravella que prové

D’esprit que fins aquí llença claror.

I tot el cel, al qui no manca re

Sinó haver-lo, demana'l al Senyor,

I tots els Sants ne volen la mercè:

Només pietat ens dóna la raó.

Que Deu oint que's parla d'ella: «Amats»

-Diu an els sants — «no vos despacienteu,

Que allà on ella és hi ha algú que quan l'haureu

A l'infern anirà a dir als condemnats:

—Jo he vist la esperança dels benaventurats—».

Ma dona és desitjada a dalt del cel!

Ara os diré quina és la sua virtut:

Dona que vulla apendre gentilesa,

Que vaja amb ella, que quan va fent via

En els homes grollers posa una feresa

Que aquell que baixament la miraria

$\mathrm{O}$ en seria ennoblit o's moriria.

Mes quan ne troba algun que digne sia

De mirar-la, en aquell té tal virtut

Que se sent molt mellor de lo que's pensa,

I tan humil, que oblida tota ofensa.

I encara major gracia Déu li ha dat:

Que no pot mal finir qui li ha parlat.

Color de perla, que a bell rostre escau,

És el seu; esblaimat, però no massa;

És tot lo bé que puga fer natura

I d'humana bellesa la mesura.

I dels ulls van sortint-li mentres passa

Uns esperits d'amor tots inflamats

Que fereixen els ulls del qui la mira

I van de dret al cor; i dur estragit

Amor meteix al rostre: tal el gira

Que no pot pas mirar-se fit a fit.

\section{Analisi della traduzione}

La versione di Maragall sembra scritta precipitosamente, a far un piacere all'amico Rahola e agli altri membri della rivista. Per cui, il Poeta non si perita, dopo un dubbioso tentativo iniziale nel frammento della prima strofa, di riprodurre lo schema metrico dell'originale (ABBC ABBC, CDD CEE), ma dà alla traduzione una struttura rimica eccentrica non riconducibile ad alcuna regola fissa. Dubbioso si è detto, in quanto anche nei primi versi c'è un bisticcio di rime all'occhio -ór, -ir, -ir-, òr; ór, -ir, -ir, òr. Oltretutto, a chiudere il fram- 
mento è, nella sirma, la stessa parola, cor, che era alla fine della fronte, senza spostamento di significato. ${ }^{18}$ In questa fase, tranne per l'ultimo sintagma in rima, la versione è quasi calcata all'originale.

Ma nei versi successivi Maragall si discosta dal testo dantesco, manifestando una certa insofferenza. Si va dalla rima, che trova soluzioni bizzarre, fino al computo sillabico che, pur mantenendo la base endecasillabica dell'originale, si deforma alla fine della seconda strofa in un verso ipermetro (v. 21); del resto, se non sospettoso anch'esso d'ipermetria, è quantomeno appesantito da sineresi e sinalefi il v. 19. In questo caso, giustificare i difetti giocando a rimpiattino dietro il concetto della parola viva è operazione che va senz'altro scartata. Lo dimostra, proprio nella strofa in esame, una svista di Maragall che, di fronte ai v. 24-25 di Dante, si confonde durante la stesura per cui, scrivendo il secondo emistichio dell'ultimo verso ripete il precedente e infine, consapevolmente o meno, li riaccorpora in un unico verso. In ultimo, la rima raó di questa strofa potrebbe aver condizionato, retrospettivamente, la sostituzione del primo emistichio del v. 4 della precedente, dove era stato piazzato inizialmente un raonar; ma, in quel caso, bisognerebbe ammettere che l'Autore non si sia accorto che, così facendo, raddoppiava, invece, il parlar nella stessa strofa. ${ }^{19}$

18. Secondo Camps, il testo di Maragall produce "5 estrofas irregulares de 8, 8, 6, 12 y 10 versos cada una, con una cierta regularidad de rima que no alcanza, ni de lejos, la rotundidad de la canción de Dante. Así, en la primera, hallamos: ABBAABBA; en la segunda: ACCACACA; en la tercera: DEEDDE; en la cuarta: FGHGHHHFIIJJ; y en la quinta y última: KLMMLDNONO, de tal modo que se recupera una rima anterior de la tercera estrofa en -ats»; cfr. A. CAMPS, "De una canción de La Vita Nova...", op. cit., p. 10. Lasciando perdere ogni altra considerazione, tra cui anche il computo delle strofe per cui si rimanda alla nota seguente, ci si concentri sulla lettura dello schema metrico della prima stanza. Camps sostiene la riduzione di -òr e -ór a un unico elemento. In epoca prefabriana, si sa, era un espediente generalmente accettato e si potrebbero addurre a dimostrazione anche esempi verdagueriani. Tuttavia, qui diverrebbe inutilmente azzardato allontanare la traduzione dall'originale quando, proprio in questa prima strofa, è riscontrabile senza forzature una perfetta corrispondenza.

19. Nel testo riprodotto da Fulcarà l'errore più grave di trascrizione è all'altezza di questo finale di strofa. Fulcarà salta un verso e mantiene nella stessa stanza, come pubblicato nella rivista, quello che era in realtà il primo verso della strofa seguente, producendo un effetto quasi comico: "Al infern anirá á dî als condemnats: / Ma dona es desitjada á dalt del cel!». Camps, invece, recupera il v. 21, «-Jo he vist la esperança dels benaventurats», ma giustifica la collocazione anticipata del v. 22, aggiungendovi peraltro una congiunzione ("I ma dóna...»). Se quest'ultimo errore può essere considerato come un semplice refuso, Camps toglie, con il suo commento, ogni dubbio riguardante l'intenzionalità del resto della sua trascrizione: «los sutiles cambios en la puntuación y, muy especialmente, en la distribución estrófica, por ejemplo el traslado del verso 28 -inicio de la estrofa 4 en Dante y conclusión de la 3 en Maragall- sugieren una acentuación diferente de la canción de Dante. Acentuación que se incremente con ese "cel!» exclamativo en posición final que remata la estrofa 3 de un modo muy fuerte»; cfr. A. CAMPS, "De una canción de La Vita Nova...», op. cit., p. 10. Infatti, 1) non si sa sull'appoggio di quali basi, Camps sdoppia la seconda stanza generandone una nuova che comincia con "Que Déu ohint...»; 2) tutta la pomposa prolusione centrata sulla parola cel in esclamazione cade di fronte alla restituzione del verso in questione alla sua legittima strofa. È vero che nel manoscrito c'è uno spazio piuttosto esteso tra questo verso e il seguente, ma da questa constatazione all'idea di una divisione strofica — oltretutto collegando il verso a inizio pagina con quello finale della pagina precedente — c'è lo stesso passaggio che porta dalla congettura all'illazione. 
Anche nella terza strofa c'è un «risparmio» per assimilazione di contenuti, per cui il secondo piede della fronte si riduce a tre versi. Si perde la specificazione che il "qual» del distico finale della fronte rappresenta rispetto ai "cor villani» (un piccolo gruppo ritagliato all'interno di un insieme più vasto di persone), per cui la relazione fra gli homes grollers e l'exemplum segnato da que aquell que è divenuta in catalano un rapporto di comparazione e il primo que va letto con il senso di «tanto che, al punto che». L'oíen che principia il v. 28 , va inteso, naturalmente e alla luce del manoscritto, come un semplice refuso. Difficile stabilire se sia da collegare a questa parola o alla collocazione anticipata del v. 22, il seguente commento spassionato di Maragall, evidentemente in risposta a delle scuse precedenti:

Jo estic molt content de que m'hagin volgut a tan bona companyia. Em sembla que no cal donar fe de l'errada tipogràfica: de sentit natural s'esmena tota sola, em sembla. De tots modos vostès facin com els sembli millor. ${ }^{20}$

La quarta strofa della Canzone è l'ultimo brano tradotto, frammentariamente, da Maragall, che ricusa quindi lo sforzo di cimentarsi con il congedo. Viene ovviamente da pensare — ma è impossibile provarlo — che le ragioni siano ideologiche, data la tipicizzazione della formula. In questo caso, la versione catalana presenta una duplicazione con uno scambio di posizioni rispetto all'originale. Se Maragall ha un colpo d'orgoglio che fa risplendere il suo primo verso (poi erroneamente anticipato, come si è già detto), cade nella ridondanza con quell'esblaymat, però no massa che sembra più che altro una glossa per facilitare la spiegazione, mentre l'inversione del rapporto "misura/natura» si presenta come un'illuminazione molto più felice. Quindi, Maragall evita il verso, forse per lui troppo dottrinale, "per esempio di lei beltà si prova»: sarà del tutto casuale, ma ancora una volta è il secondo piede della fronte ad essere ridotto a tre versi.

Particolarmente interessante è però il risultato dell'ultimo sforzo maragalliano. Il movimento degli occhi del testo italiano viene inserito in un contesto di movimento globale della persona. Un dî estragit viene infine attribuito a un'azione d'Amore, pensato come immagine quasi immobile nell'originale e anche l'impossibilità di guardare è applicata a un oggetto in movimento di cui non è chiara la natura: tal el gira, infatti, può essere retto o da Amore o dalla persona di lei - e non ci aiuta la prima versione del manoscritto che riportava un aixis es dit che fungeva chiaramente da riempitivo.

\section{Conclusioni}

Letta nella sua globalità, la versione è piuttosto irregolare e raffazzonata, anche se risplende in alcuni punti di luce propria — specialmente se si è predisposti ad accettare certe forzature d'autore. Le varianti del manoscritto sono poche 
e il testo sembra essere stato steso di getto. Si obietterà che le brutte copie di Maragall sono sempre molto pulite e quasi definitive, ma resta il sospetto di un'intepretazione forzata basata su testi che non possono essere definiti come abbozzi. E, forse, in fondo, aveva ragione Garcès quando replicava:

L'esmena, el retoc, la tria dels mots, l'obediència a una memòria i una música pregones, són normals en el moment de la creació del poema. Tothom sap que fins en aquells autors més senzills i entenendors com, posem per cas, Verdaguer, o en els més obstinats fidels de la "paraula viva» i a llur davant Maragall, trobaríem els manuscrits plens d'eliminacions i d'afegits successius, palpitant de dubte que ens mostren quins viaranys abruptes han calgut seguir a remolc de la inspiració $[\ldots]^{21}$

D'altra parte, l'impegno di Maragall sul piano concettuale, sin dalla corrispondenza intelletto/enteniment, sembra essersi profuso in un rispetto dell'originale dietro il quale fa capolino la devozione del Modernismo verso il testo della Vita Nuova. ${ }^{22}$

Non si insisterà ora sugli aspetti più complessi che legano Maragall all'opera di Dante. É invece il caso di sottolineare, una volta per tutte, l'importanza di questa traduzione dantesca pubblicata in vita: in un'ottica al tempo stesso specifica e generale sarà giocoforza ammettere il rapporto che le traduzioni in catalano di Maragall mantengono con il suo percorso di ricerca letteraria. Dal Faust alle Elegie romane, dagli Inni omerici alla Prima Olimpica di Pindaro, dal Così parlò Zarathustra al Tristano e Isotta di Wagner, passando per l'Enrico di Ofterdingen di Novalis, le versioni vengono intraprese in base a una serie di scelte strategiche e estetiche che evidenziano le influenze più profonde e varie che si stratificano nell'opera di Maragall. Ma il Nostro ha la straordinaria capacità di tesserle minuziosamente insieme, di adattarle e di inglobarle nella propria poetica, impedendo quel corto circuito che sarebbe logico aspettarsi da risultati e programmi così eterogenei. ${ }^{23}$

21. Cfr. Tomàs GARCÈs, «El rigor poètic de Josep Carner», in ID., Sobre Salvat-Papasseit i altres escrits, Barcellona: Selecta, 1972. Ora in ID., Prosa completa II, a cura d'Àlex SuSANNA, Barcellona: Columna, 1991, p. 327.

22. Cfr. almeno Maria-Àngela CERDÀ I SURROCA, Els pre-rafaelites a Catalunya. Una literatura i uns simbols, Barcellona: Curial, 1981.

23. Ė doveroso ringraziare la bibliotecaria responsabile dell'Arxiu Maragall, Dolç Nom de Maria Tormo i Ballester, che si è prodigata in utilissimi aiuti, consigli e suggerimenti. 
Vita Nuova, XIX. Intorno a una traduzione di Joan Maragall Quaderns d'Italià 8/9, 2003/2004 207

Hax dom os desitind a daetder cel!. Are os ine guina es h rua virtul: om pore mille opentre gentiter

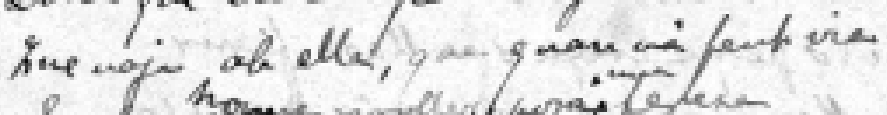

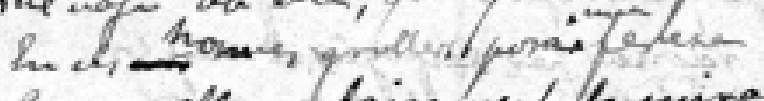

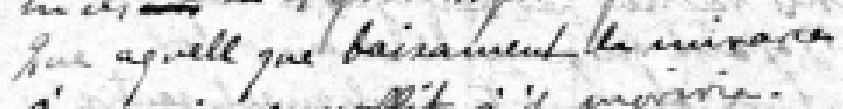

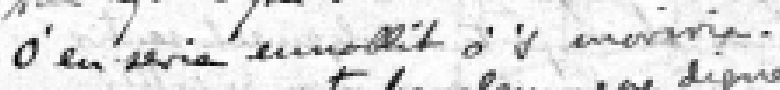

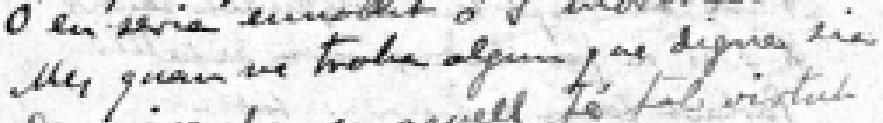

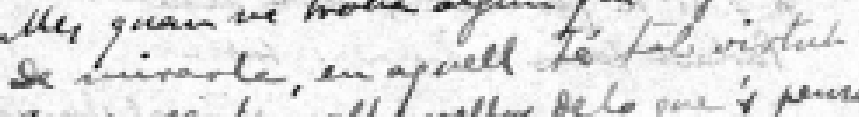

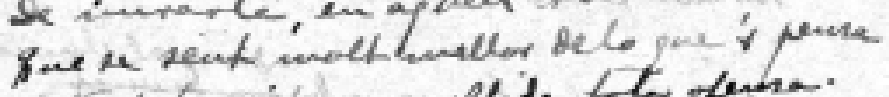
1 tant tumil pare oblide tL fenna: tevere myit macie des l'he dat the so pot had finis quil i bi partat

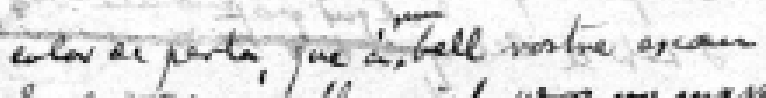

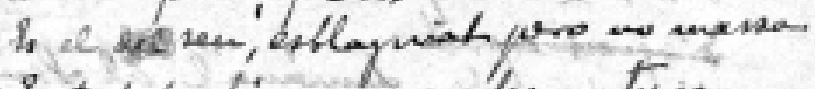

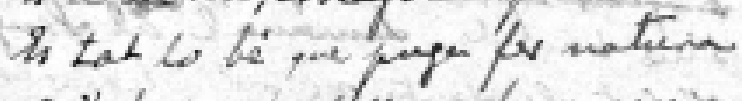

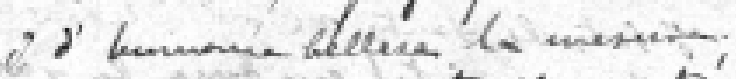

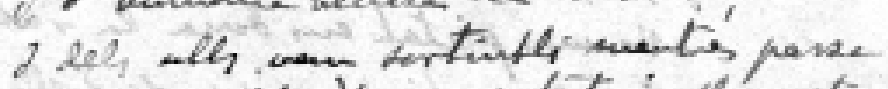

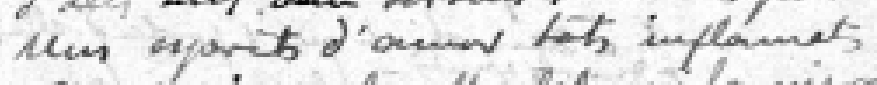

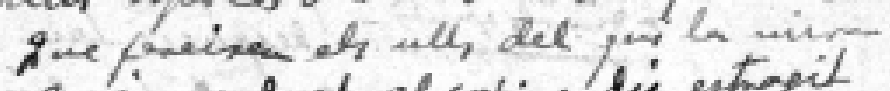

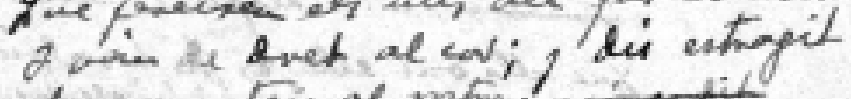

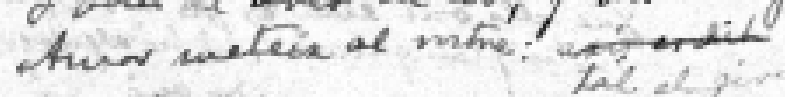
greng potpar wivine $f x-f i t$

Manoscritto autografo della traduzione di Maragall della Vita Nuova. Arxiu Maragall. 
208 Quaderns d'Italià 8/9, 2003/2004

Francesco Ardolino

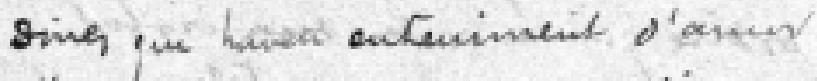
Vull at inative, hels mero dir ivo pergue crega ou llaor finir

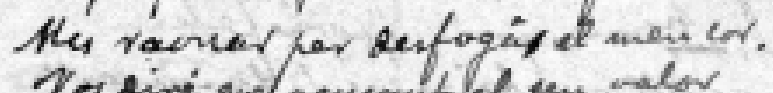

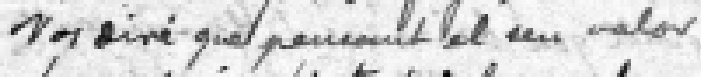
Arua de in pi tat dolgament sentir

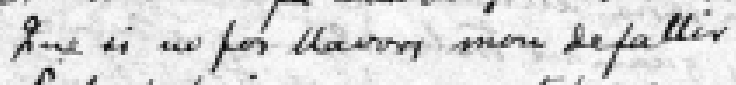
forlant bario manower fat cor

Un angel lame ob givinal daner $7 \mathrm{hin}$ "Sanger, al man othi endevé the gran answatis que preané s'eoprit, we firs of wi vene elams

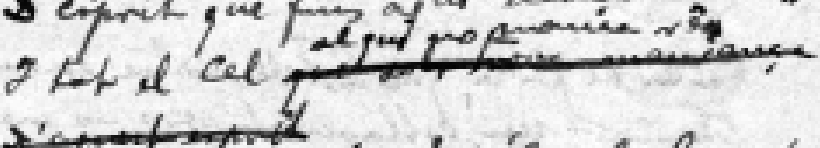

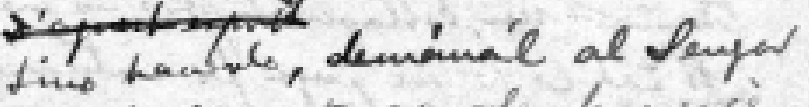

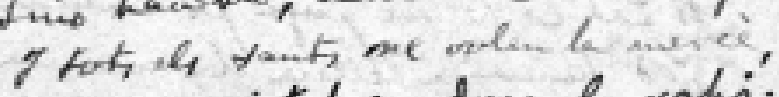
Wo mes pietal an done \& rahi:"

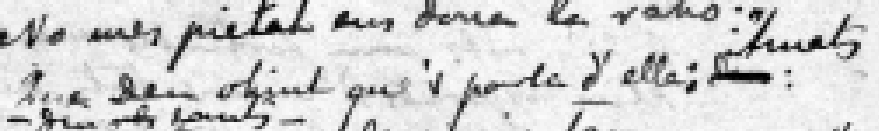
- gi th tons in Respheiantan

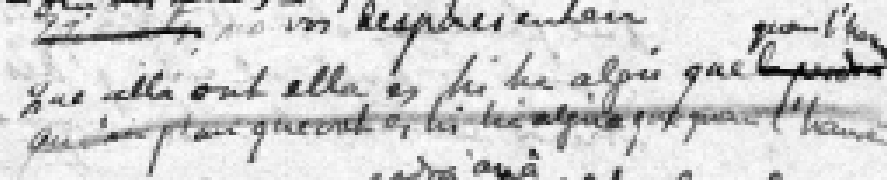

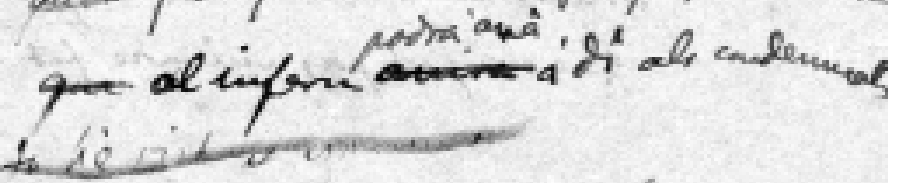

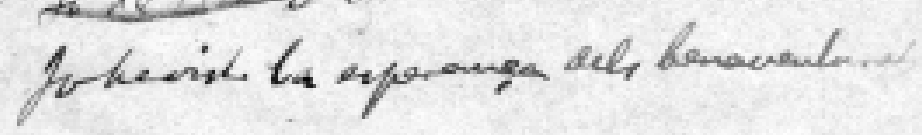

